\title{
Chair's Letter: A Great Opportunity
}

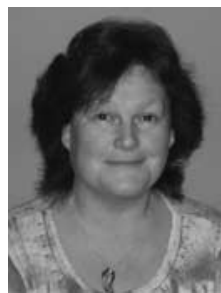

Manda Dunne

BARNA Chair

Dear BARNA members,

$\mathrm{H}$ aving just returned from Ljubljana, Slovenia where I attended the 2012 WCNA Congress (World Congress of Nurse Anaesthetists), it is with great pride that I can tell you BARNA (British Anaesthetic and Recovery Nurses Association) has won the bid to host the 2016 WCNA in Glasgow. This is a great opportunity for us to raise the profile of Anaesthetic Nurses in the United Kingdom and to leave a lasting legacy to the profession of anaesthetic and recovery nursing. I want to assure you that this congress will include both anaesthetic and recovery topics.

You may be interested to know that in the IFNA (International Federation of Nurse Anaesthetists) Educational Standards for Preparing Nurse Anaesthetists it says: 'Nurse Anaesthetist' - This is a general title for nurses working in anaesthesia with a specific education.

The WCNA congress has never been held in the United Kingdom before, so I feel very privileged that we have this opportunity. I hope that you will be able to support us and make the journey to Glasgow to attend the congress in 2016. Four years may seem like a long way away at the moment, but believe me it will be here before we know it!

The keynote speech, delivered by Polona Selic, was entitled Anaesthetic Nurses: 'Stepping towards a New Professional Development?' This was very interesting and opened up the discussion about the special emphasis on research in the nursing profession, the need for professional identity, career structures and pathways as well as professional role modification. In turn, this is offered as a contribution to establishing an active nursing research culture and promotion of evidence-based practice.

Also, many countries are anticipating a shortfall in the supply of Anaesthetists, so they are exploring working models in other countries that currently work with fewer physicians and more nurses. It is very interesting to look at the history in various countries and see how the role of the Nurse Anaesthetist came about, especially in the United States of America. Also, in many third world countries there are so few physicians that the nurse is often alone and has no choice but to administer anaesthesia unsupervised as there is no alternative.

The WHO European Strategy for Continuing Education for Nurses and Midwives 2003 declared that Anaesthetic Nurses 'require a well developed knowledge base, along with specialist skills in both technological and the caring dimensions of anaesthetic nursing, in order to meet the complex needs of patients who are undergoing surgery and anaesthesia'. It is interesting, therefore, that there has been an ongoing process of re-evaluating the practice of anaesthetic nurses due to confusion surrounding accountability, especially when working in an 'extended role' between the medical and nursing profession. I relate this as a worldwide example incidentally. Is it that one of the most significant obstacles could be the difficulty in assessing competency in professionalism and that we should be aiming at a deeper understanding of teamwork and the role of the nurse with a patient centred, holistic health provision?

This leads me to the conclusion that hosting a World Congress of this size is such a wonderful forum to network with colleagues from all corners of the world and to share in education that will provide something for everyone, and an opportunity to form lasting links with each other, in the words of Benjamin Franklin:

'We must, indeed, all hang together or assuredly we shall all hang separately'.

Thank you. Let's look forward to 2016 together. 